\title{
ON THE BEHAVIOR OF THE LINC TRANSMITTER
}

\author{
Fernando CASADEVALL, Juan J. OLMOS
}

Departament de Teoria del Senyal i Comunicacions

Universitat Politecnica de Catalunya

Apdo. 30002, 08080 Barcelona, SPAIN

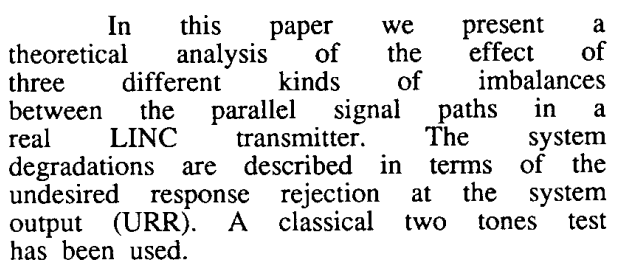

\section{INTRODUCTION}

In mobile radio systems, the relatively inefficient use of the spectrum by the present FM-type modulations, like MSK, TFM, etc... has manifested itself as crowding on the available channels. However, they are still widely used because their constant envelope property is appropriate for using power efficient non linear amplifiers. In the most recent studies of digital mobile radio systems, the introduction of linear modulation methods, such as QPSK and M-QAM, is considered in order to improve spectrum efficiency. However, as M-QAM has a
non-constant envelope, it will be non-constant to consider the linear power amplifiers, which are less efficient that the classical class-C power amplifiers used with the FM-type modulations.

In order to achieve both spectrum and power efficiency, several techniques of linearizing power amplifiers have been presented in the literature, [1], [2], [3], [4]. Among all of them, one of the more promising appears to be the so called LINC transmitter. The basic principle of the LINC transmitter is to represent any arbitrary bandpass signal, which may have both amplitude and phase variations, by means of two signals which are of constant amplitude and have only phase variations. These two angle modulated signals can be amplified separately using efficient high-power non linear devices. Finally the amplified signals are passively combined to produce an amplitude modulated signals.

\begin{abstract}
In a practical LINC transmitter there are three main mechanisms that degrade the overall performance: the power gain and delay (or phase) imbalance between the two RF paths and the different non-linear characteristics of both RF power amplifiers. Even though some practical implementations have pointed out the effects of these degradations in the system performances, with respect to the theoretical behavior there is not yet, to our knowledge, a closed expression providing a characterization of these effects.

In this paper we present a theoretical analysis of the effect of the above mentioned imbalances between the parallel signal paths in the system performance. The system degradations are described in terms of the undesired response rejection at the system output (URR). A classical two tones test has been used.
\end{abstract}

\section{SYSTEM DESCRIPTION}

Figure 1 shows the schematic drawing of LINC system, where:

$S(t)=a(t) \cdot \cos \left[\omega_{0} t+\theta(t)+\phi\right]$

$S_{1}(t)=V / 2 \cdot \sin \left[\omega_{0} t+\theta(t)+\psi(t)+\phi\right]=$

$=S_{I 1}(t) \cos \left[\omega_{0} t+\phi\right]+S_{Q 1}(t) \sin \left[\omega_{0} t+\phi\right]$

$S_{2}(t)=V / 2 \cdot \sin \left[\omega_{0} t+\theta(t)-\psi(t)+\phi\right]=$

$=S_{I 2}(t) \cos \left[\omega_{0} t+\phi\right]+S_{Q 2}(t) \sin \left[\omega_{0} t+\phi\right]$

with:

$\psi(t)=\sin ^{-1}[a(t) / V], \quad$ being $\quad \max [a(t)] \leq V$ and: 


$$
\begin{aligned}
& S_{I 1}(t)=\frac{1}{2}\left[a(t) \cos \theta(t)+\sin \theta(t) \sqrt{V^{2}-a^{2}(t)}\right] \\
& S_{Q 1}(t)=\frac{1}{2}\left[-a(t) \sin \theta(t)+\cos \theta(t) \sqrt{V^{2}-a^{2}(t)}\right] \\
& S_{12}(t)=\frac{1}{2}\left[-a(t) \cos \theta(t)+\sin \theta(t) \sqrt{V^{2}-a^{2}(t)}\right] \\
& S_{Q 2}(t)=\frac{1}{2}\left[a(t) \sin \theta(t)+\cos \theta(t) \sqrt{V^{2}-a^{2}(t)}\right] \\
& \text { operations are required only when the } \\
& \text { signals } S_{I 1}(t), S_{Q 1}(t), S_{12}(t), S_{Q 2}(t) \text { are } \\
& \text { generated. As this signals only depend on } \\
& \text { a(t) and } \theta(t), \text { which are baseband signals, } \\
& \text { the non linear operations could be easily } \\
& \text { performed using a Digital Signal Processor } \\
& \text { (DSP). Then, we only need to use a DSP, } \\
& \text { four balanced modulators, and two } \\
& \text { combiners to generate the signals } S_{1}(t) \\
& \text { and } S_{2}(t) \text {. }
\end{aligned}
$$

\section{SYSTEM IMPAIRMENTS}

In a practical LINC amplifier there are three main mechanisms that degrade the overall performance:

\section{A: Imbalance between the path gains}

In this case :

$S(t)=G \cdot S_{1}(t)-(G+\Delta G) \cdot S_{2}(t)=$

$=G a(t) \cos \left[\omega_{0} t+\theta(t)+\phi\right]$ -

$-\Delta G \frac{V}{2} \sin \left[\omega_{0} t+\theta(t)-\Psi(t)+\phi\right]$

that is, a residual level of the one of the phase modulated signals appears in the system output.

In order to analyze the effect of this undesired signal on the system performance, we consider the classical two tone test. In this case: $a(t)=2 A \cos \left(\omega_{m} t\right)$ and $\theta(t)=0$. Considering that $\phi \equiv 0$ (with no loss in generality) the useful output signal is :

$$
S_{U}(t)=G\left[A \cos \left(\omega_{1} t\right)+A \cos \left(\omega_{2} t\right)\right]
$$

with $\omega_{1}=\omega_{0}+\omega_{m}$ and $\omega_{2}=\omega_{0}-\omega_{m}$. The interfering signal is :

$$
\begin{aligned}
& S_{I}(t)=-\Delta G \frac{V}{2} \sin \left[\omega_{0} t-\psi(t)\right]= \\
& =\frac{\Delta G}{2}\left[A \cos \left(\omega_{1} t\right)+A \cos \left(\omega_{2} t\right)\right]- \\
& -\frac{\Delta G}{2} \sqrt{V^{2}-\left[2 A \cos \left(\omega_{m} t\right)\right]^{2}} \sin \left(\omega_{0} t\right)
\end{aligned}
$$
as outside the desired bandwidth.

$$
\text { The most important of all the }
$$
interfering spectral lines is placed at $\omega_{0}$. Its level is given by:

I.L. $=\frac{2}{I} \frac{\Delta G}{2} V \int_{0}^{\pi / 2} \sqrt{1-A_{x}^{2} \cos ^{2}(\vartheta)} d \vartheta=$

$=\frac{2}{\Pi} \frac{\Delta G}{2} \mathrm{VE}\left(\mathrm{A}_{\mathrm{x}}, \pi / 2\right)$

with $A_{x}=2 A / V \leq 1, \quad \vartheta=\omega_{m} t$, and $E(x, y)$ being the elliptic integral of the second kind, [5].

Then, considering that the level of the useful spectral line is $A(G+\Delta G / 2) \cong A G$, we can define the URR at the output as:

$U R R(d B)=-20 \log \left[\frac{\Delta G}{G}\right]-20 \log \left(\frac{2 E\left(A_{x}, \pi / 2\right)}{\prod_{x} A_{x}}\right)$

\section{B: Imbalance between the path delays}

If the two paths have different delays, the signals, at the input of the combiner are not in phase, and the resultant signal shows a high degree of distortion. We now consider that:

$$
\mathrm{S}(\mathrm{t})=\mathrm{G} \cdot \mathrm{S}_{1}(\mathrm{t})-\mathrm{G} \cdot \mathrm{S}_{2}(\mathrm{t}-\tau)
$$

where $\tau$ can be expressed as $\tau=\gamma / \omega_{0}$. After some algebraic operations, the above expression could be written as :

$S(t)=G a(t) \cos \left(\omega_{0} t\right)+$

$+G[1-\cos (\gamma)] S_{2}(t)+G \sin (\gamma) S_{3}(t)$ 
with

$\mathrm{S}_{3}(\mathrm{t})=V / 2 \cdot \cos \left[\omega_{0} t-\psi(\mathrm{t})\right]=$

$=V / 2 \cdot \sin \left[\omega_{0} t-\psi(t)+\pi / 2\right]$

Comparing the expressions of $\mathrm{S}_{2}(\mathrm{t})$ and $S_{3}(t)$ it is obvious that both signals have equal power spectrum. So, considering

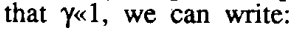

$S(t) \cong G a(t) \cos \left(\omega_{0} t\right)+G \gamma S_{3}(t)$

As before, assuming that $a(t)=2 A \cos \left(\omega_{m} t\right)$, and considering that $\mathrm{S}_{3}(\mathrm{t})$ is a phase modulated signal, the most relevant of all the interfering spectral lines is placed at $\omega_{0}$ and its level is given by :

$$
\text { I.L. }=\frac{\mathrm{G} \cdot \gamma \cdot \mathrm{V}}{\mathrm{I}} \mathrm{E}\left(\mathrm{A}_{\mathrm{x}}, \pi / 2\right)
$$

with $A_{x}=2 A / V$. The URR can now be expressed as:

$\mathrm{URR}(\mathrm{dB})=-20 \log (\gamma)-20 \log \left(\frac{2 \mathrm{E}\left(\mathrm{A}_{\mathrm{x}}, \pi / 2\right)}{\Pi \mathrm{A}_{\mathrm{x}}}\right)$

C: Imbalance in the non-linear characteristics of power amplifiers

The non-linear behavior of the power amplifiers can be characterized by means of the following expression :

$$
v_{0}(t)=a v_{i}(t)+b v_{i}^{2}(t)+c v_{i}^{3}(t)
$$

where "a" is the gain for low signal level. In this case, the input signal is $v_{i}(t)=S_{1}(t)$ or $v_{i}(t)=S_{2}(t)$. Then, defining $\mathrm{v}_{01}(\mathrm{t})$ (resp. $\mathrm{v}_{02}(\mathrm{t})$ ) as the output signal, it results in :

$$
\begin{aligned}
& v_{01}(t) \cong a\left[1+\frac{3 c_{1}}{4 \frac{a}{2}}\left(\frac{v}{2}\right)^{2}\right] S_{1}(t) \\
& v_{02}(t) \cong a\left[1+\frac{3 c_{2}}{4 \frac{a}{2}}\left(\frac{V}{2}\right)^{2}\right] S_{2}(t)
\end{aligned}
$$

In the above expressions $c_{1}$ (resp. $c_{2}$ ) denotes the third order characteristic of the first (resp. second) non-linear power amplifier. $c_{1}$ and $c_{2}$ are negative constants.

From $v_{01}(t)$ and $v_{02}(t)$, the output signal $S(t)$ can be written as :

$$
\begin{aligned}
S(t)= & v_{01}(t)-v_{02}(t)= \\
= & a\left[1+\frac{3 c_{1}}{4 a}\left(\frac{V}{2}\right)^{2}\right] a(t) \cos \left(\omega_{0} t\right)+ \\
& +\frac{3}{4}\left(\frac{V}{2}\right)^{3} \Delta c \sin \left[\omega_{0} t-\psi(t)\right]
\end{aligned}
$$

where $\Delta \mathrm{c}=\mathrm{c}_{1}-\mathrm{c}_{2}$. Considering again a two tones test, the interfering signal generates a set of undesired spectral lines, the most important being placed at $\omega_{0}$. This line has a level given by :

$\mathrm{I} . \mathrm{L} .=\frac{2}{\Pi}\left[\frac{3}{4}\left(\frac{\mathrm{V}}{2}\right)^{3} \Delta \mathrm{c}\right] \mathrm{E}\left(\mathrm{A}_{\mathrm{x}}, \pi / 2\right)$

Then, considering that the level of the useful spectral line is:

$$
\text { A a }\left[1+\frac{3 \mathrm{c}_{1}}{4 \mathrm{a}}\left(\frac{\mathrm{V}}{2}\right)^{2}\right] \cong \mathrm{A} \mathrm{a}
$$

The URR is:

$$
\begin{aligned}
& \operatorname{URR}(\mathrm{dB})=-20 \log \left[\frac{\Delta \mathrm{c}}{\mathrm{c}_{1}}\right]-20 \log \left(\frac{2 E\left(\mathrm{~A}_{\mathrm{x}}, \pi / 2\right)}{\Pi \mathrm{A}_{\mathrm{x}}}\right)- \\
& -40 \log \left[\frac{\mathrm{V}}{2 \mathrm{I}_{\mathrm{p}}}\right] \\
& \text { where } \mathrm{I}_{\mathrm{p}} \text { is the input third order } \\
& \text { intercept point of the first amplifier. }
\end{aligned}
$$

\section{RESULTS}

In figure 2 we show the evolution of the undesired response rejection at the imbalance, $\Delta \mathrm{G} / \mathrm{G}$, using $A$ as a parameter. It is important to emphasize that the URR depends not only on $\Delta \mathrm{G} / \mathrm{G}$ but also on the relative level of the input modulating signal $2 \mathrm{~A} / \mathrm{V}$. The smaller the relative level of the input signal (A) is, the more important is the degradation of the 
system performance. Then it is convenient to be sure that the input signal is as close as possible to the maximum permissible input level.

On the other hand, we can see that a small gain difference between both paths produces a significant degradation. For example, if the gain imbalance between paths is about $1 \%$ and the relative input signal is equal to one, the output rejection decreases to $45 \mathrm{~dB}$, but when the relative input signal is only 0.25 then the output rejection is as low as $28 \mathrm{~dB}$.

In figure 3 we show the evolution of the URR behavior against the phase imbalance $\gamma$. We can observe that a small delay imbalance between both paths causes a high degree of non linear distortion. For example, a phase error between paths as low as $2^{0}$ diminishes the undesired response rejection to only $33 \mathrm{~dB}$, for the most favorable value of $A_{x}$, that is, for $A=1$.In fact, this is the most important cause of the signal distortion on the LINC transmitter.

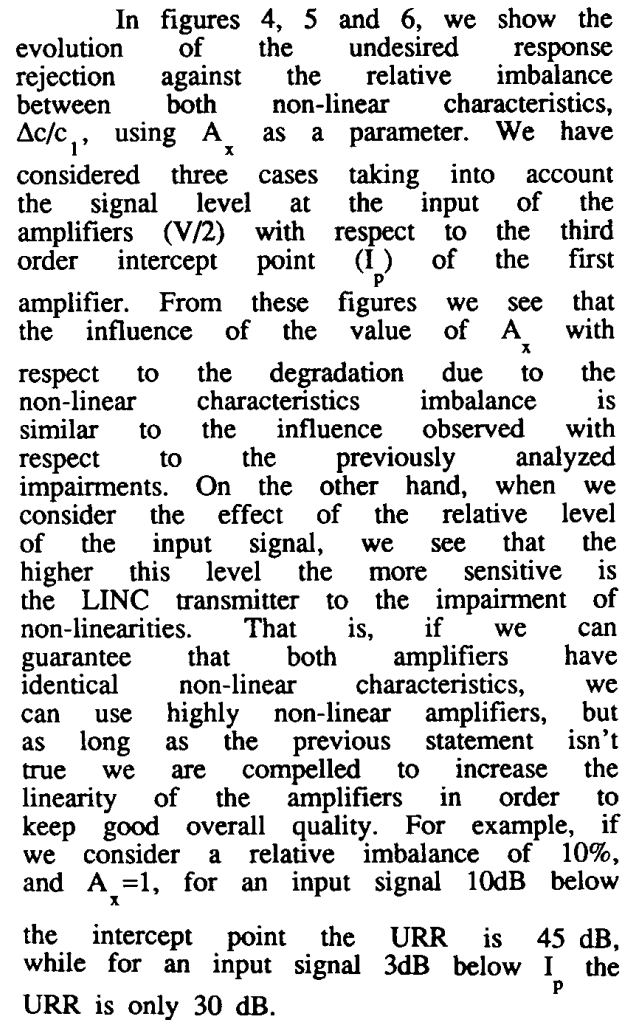

\section{CONCLUSIONS}

In this paper we have analyzed the effect of the R.F. signal processing impairments in a LINC transmitter. We have distinguished between three kinds of path imbalance. In all cases analytical expressions have been obtained in order to evaluate the effect of the imbalances on the performance of the LINC transmitter. The gain and the phase imbalance between both power amplifiers, appears as a serious constraint of the performances of the LINC transmitter. Certainly, they could produce significant reduction of the linearity of the transmitter. With respect to the imbalance between the non-linear characteristic of both power amplifiers, its effect is less meaningful even for high values of $\Delta \mathrm{c} / \mathrm{c}_{1}$ if the input signal level is kept at a reasonable margin below the $I_{p}$.

\section{ACKNOWLEDGEMENTS}

This work was supported by CICYT (Spain) under Grant TIC 880543

\section{REFERENCES}

[1] D.C. Cox, "Linear Amplification with Non-linear Components", IEEE Trans. Communications, COM-22, 1974, pp.1942-1945.

[2] Y. Akaiwa, Y. Nagata, "A Linear Modulation Scheme for Digital Mobile Radio Communications", IEEE GLOBECOM 1985, pp.965-969.

[3] Y. Nagata, "Linear Amplification Technique for Digital Mobile Communications", IEEE VTC 1989, pp.159-163.

[4] A. Bateman, R.J. Wilkinson, J.D Marvill, "The Application of Digital Signal Processing to Transmitter Linerisation", IEEE 8th European Conference on Electrotechnics, 1988, pp.64-67.

[5] M. Abramowitz, I. Stegun, "Handbook of Mathematical Functions", Dover Publishing Inc., New York, 1970. 


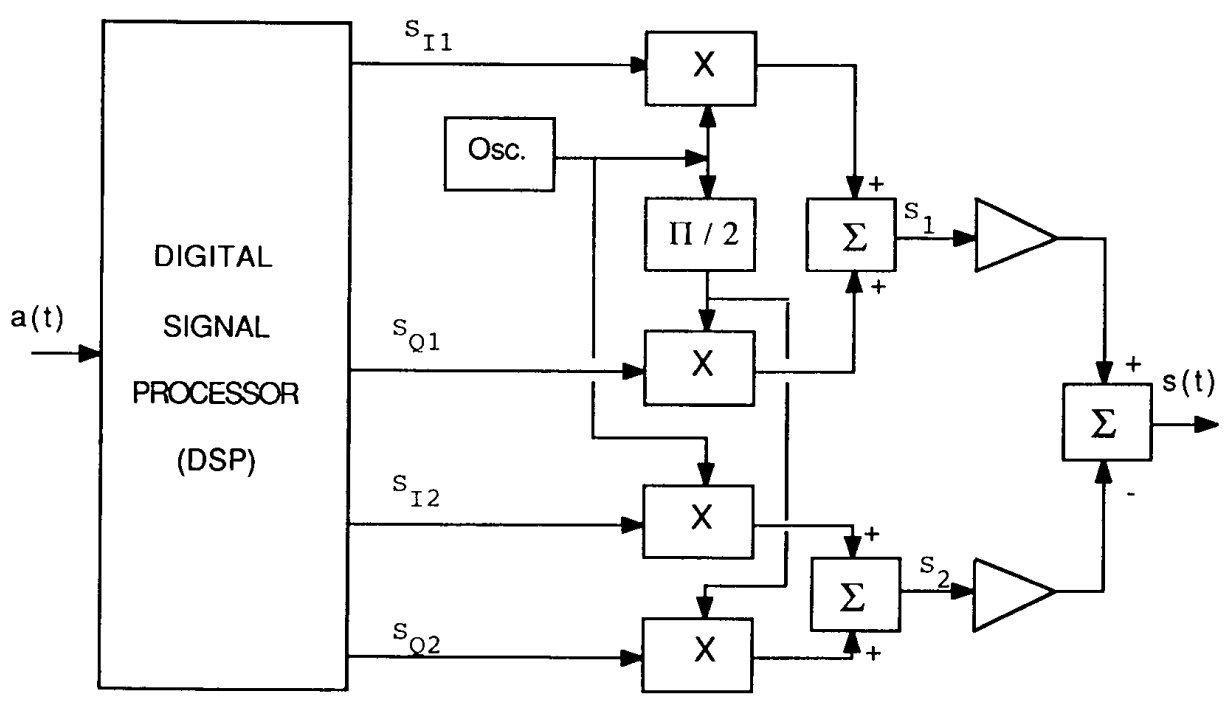

Figure 1.- Schematic Diagram of the LINC Transmitter
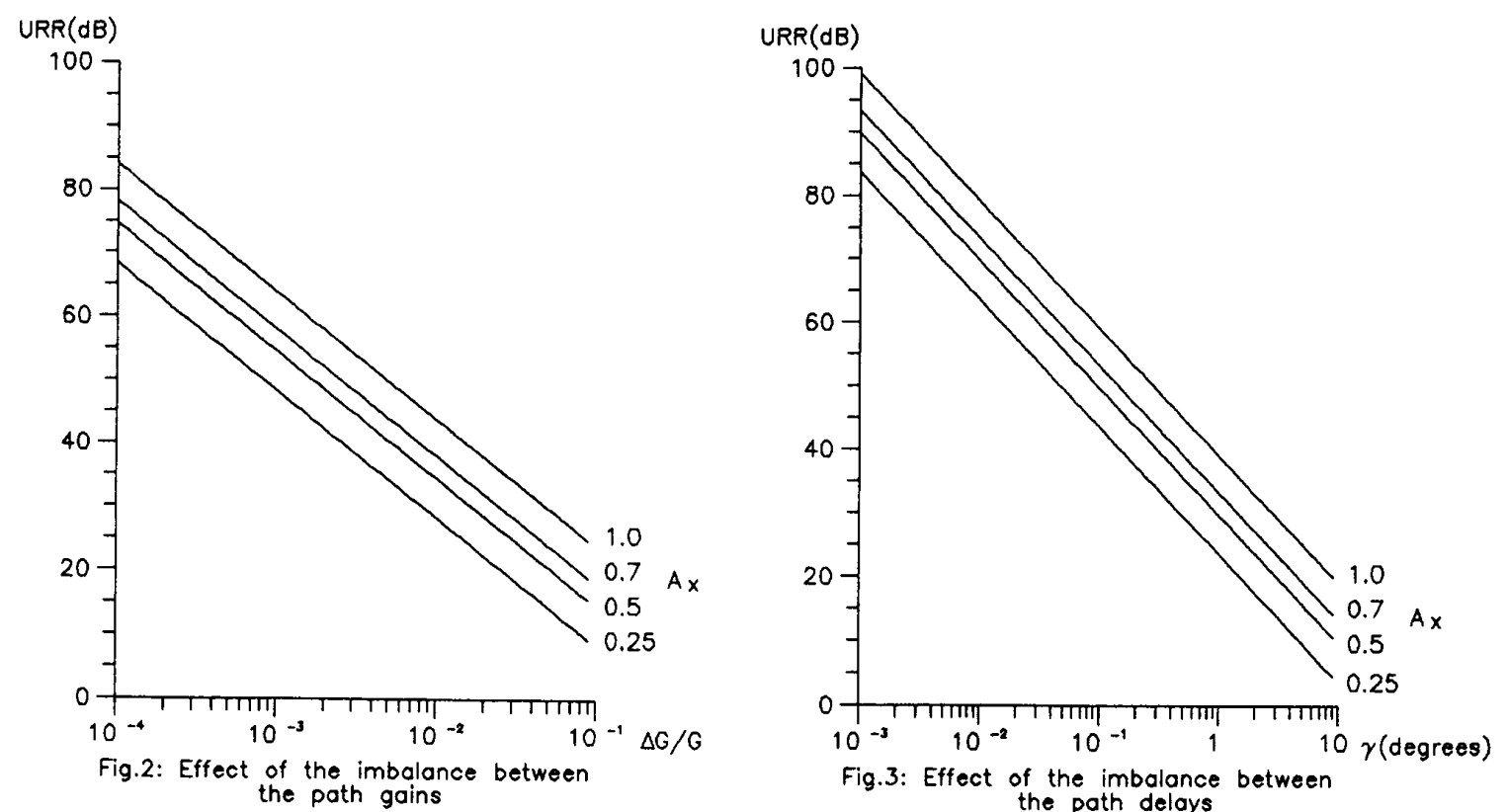

Fig.3: Effect of the imbalance between the path delays 

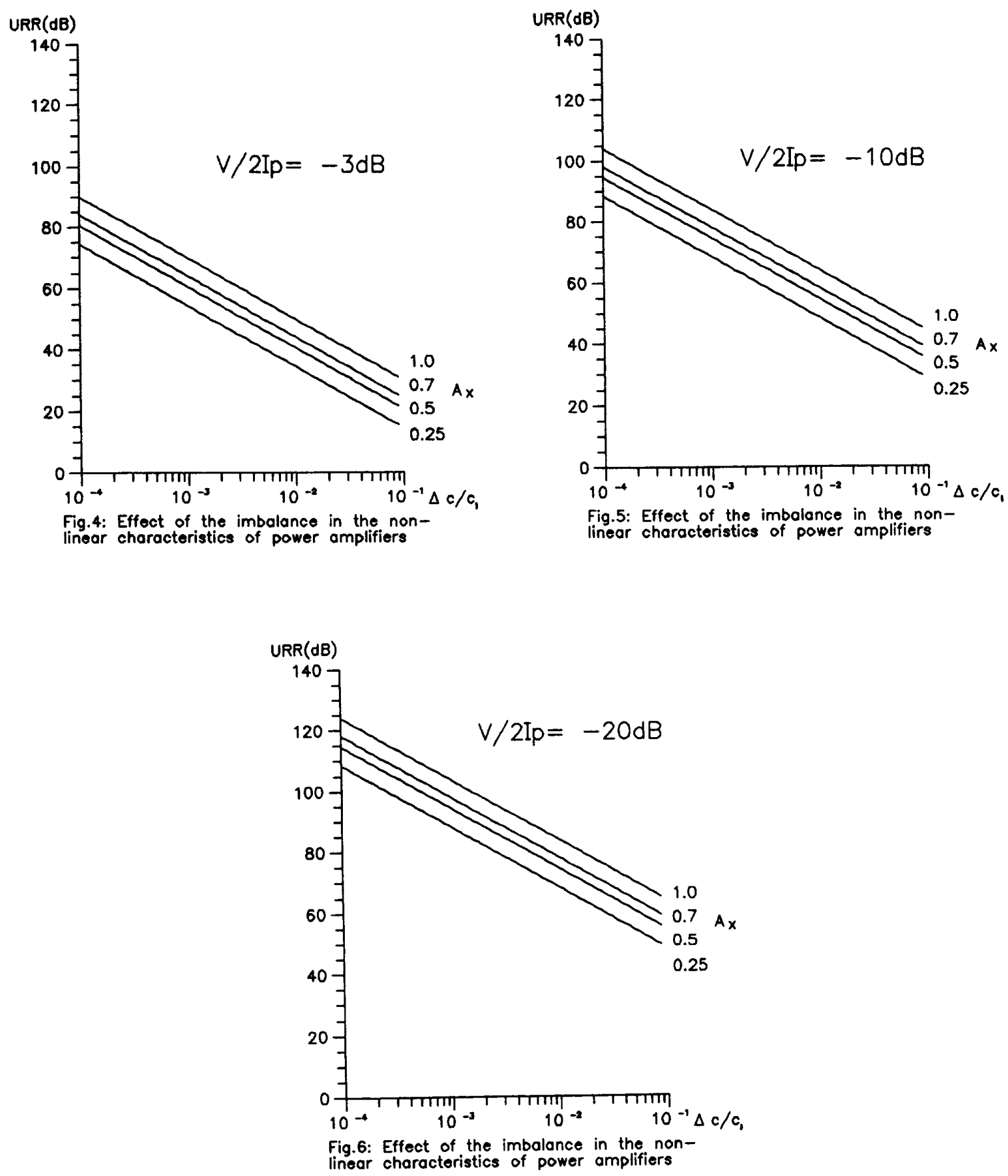